\title{
CRTC1-MAML2 gene fusion in mucoepidermoid carcinoma of the lacrimal gland
}

\author{
SARAH LINÉA VON HOLSTEIN ${ }^{1,3}$, ANDRÉ FEHR ${ }^{3}$, STEFFEN HEEGAARD $^{1}$, \\ MARIANNE HAMILTON THERKILDSEN ${ }^{2}$ and GÖRAN STENMAN ${ }^{3}$ \\ ${ }^{1}$ Institute of Neuroscience and Pharmacology, Eye Pathology Section, Faculty of Health Sciences, University of \\ Copenhagen; ${ }^{2}$ Department of Pathology, Rigshospitalet, Copenhagen, Denmark; ${ }^{3}$ Department of \\ Pathology, Sahlgrenska Cancer Center, University of Gothenburg, Gothenburg, Sweden
}

Received November 1, 2011; Accepted December 19, 2011

DOI: $10.3892 / o r .2012 .1676$

\begin{abstract}
Epithelial tumors of the lacrimal gland are histologically similar to salivary gland tumors. Here we report on a rare case of mucoepidermoid carcinoma (MEC) in a 73-year-old man with a swelling of the left lacrimal gland. The tumor had a microscopic appearance consistent with a classical low-grade MEC of the lacrimal gland. There were no signs of recurrence or metastases during a five-year followup. Using RT-PCR and FISH we demonstrated that the tumor was positive for the CRTC1-MAML2 gene fusion previously shown to be associated with in particular low-grade salivary MECs with favorable prognosis. By immunohistochemistry we showed that the majority of tumor cells, including epidermoid, intermediate and mucous producing cells, expressed the CRTC1-MAML2 fusion protein. In contrast, 15 non-MEC lacrimal neoplasm were fusion-negative. Our findings show that lacrimal MEC is not only clinically and morphologically but also genetically identical to MECs originating from other exocrine glands, including those of the lung, thyroid, cervix and salivary glands. Taken together, the present and previous studies further emphasize the fundamental biologic and genetic similarities among MECs developing from different anatomical sites and organs. Moreover, our findings indicate that the CRTC1-MAML2 fusion may be a useful diagnostic and prognostic biomarker for lacrimal MEC.
\end{abstract}

\section{Introduction}

Mucoepidermoid carcinoma (MEC) of the lacrimal gland is a rare tumor comprising about $1-2 \%$ of all lacrimal gland neoplasms $(1,2)$. Lacrimal MEC usually appears as a

Correspondence to: Professor Göran Stenman, Department of Pathology, Sahlgrenska Cancer Center, University of Gothenburg, P.O. Box 425, SE-405 30 Gothenburg, Sweden

E-mail: goran.stenman@1lcr.med.gu.se

Key words: mucoepidermoid carcinoma, lacrimal gland, CRTC1, MAML2, fusion oncogene, biomarker painless, slowly enlarging mass in the upper temporal part of the orbit, that causes proptosis and medial/downwards displacement of the eye (3). The mean age of patients at presentation is 49 years and females are more often effected than men ( $\mathrm{F}: \mathrm{M=3:2}$ ) (1). MEC is traditionally graded in low-, intermediate- and high-grade tumors (4). Low-grade MECs have an excellent prognosis and usually only require surgical excision whereas high-grade MECs have a poor prognosis despite intense treatment strategies (1). Previous cytogenetic studies have identified a $\mathrm{t}(11 ; 19)(\mathrm{q} 21-22 ; \mathrm{p} 13)$ translocation as a recurrent and tumor-type specific rearrangement in MECs of the salivary glands (5). More recent studies have shown that this translocation results in a fusion of the transcriptional coactivators MAML2 and CRTC1 (6-8). MAML2 belongs to a family of Mastermind-like, nuclear proteins that functions as coactivators for Notch receptors whereas CRTC1 belongs to a family of highly conserved CREB (cAMP response element-binding protein) coactivators. The CRTC1-MAML2 fusion encodes a chimeric protein consisting of the CREB-binding domain of CRTC1 linked to the transactivation domain of MAML2. The fusion protein activates transcription of in particular cAMP/CREB target gene $(7,9,10)$. Furthermore, clinical studies have demostrated that patients with fusion-positive MECs have a significantly better prognosis compared to those with fusion-negative tumors, thus establishing CRTC1-MAML2 as a clinically useful biomarker for MEC (11-13). Here we describe the first case of a MEC of the lacrimal gland with expression of the CRTC1-MAML2 gene fusion.

\section{Patients and methods}

Clinical history. A 73-year-old male presented with a swelling located temporally in the upper left eyelid (Fig. 1A). The patient had noticed the mass for $\sim 6$ months. Clinical examination revealed a $2 \times 1 \mathrm{~cm}$ firm slightly tender tumor at the site of the lacrimal gland. Visual acuity and eye motility was normal. A computed tomography scan showed a diffuse enlargement of the left lacrimal gland measuring $2 \times 1.5 \mathrm{~cm}$ (Fig. 1B). The tumor had a uniform appearance and diffuse and low signal intensity after contrast injection. A lateral orbitotomy was performed and the tumor was removed en bloc. There was no 


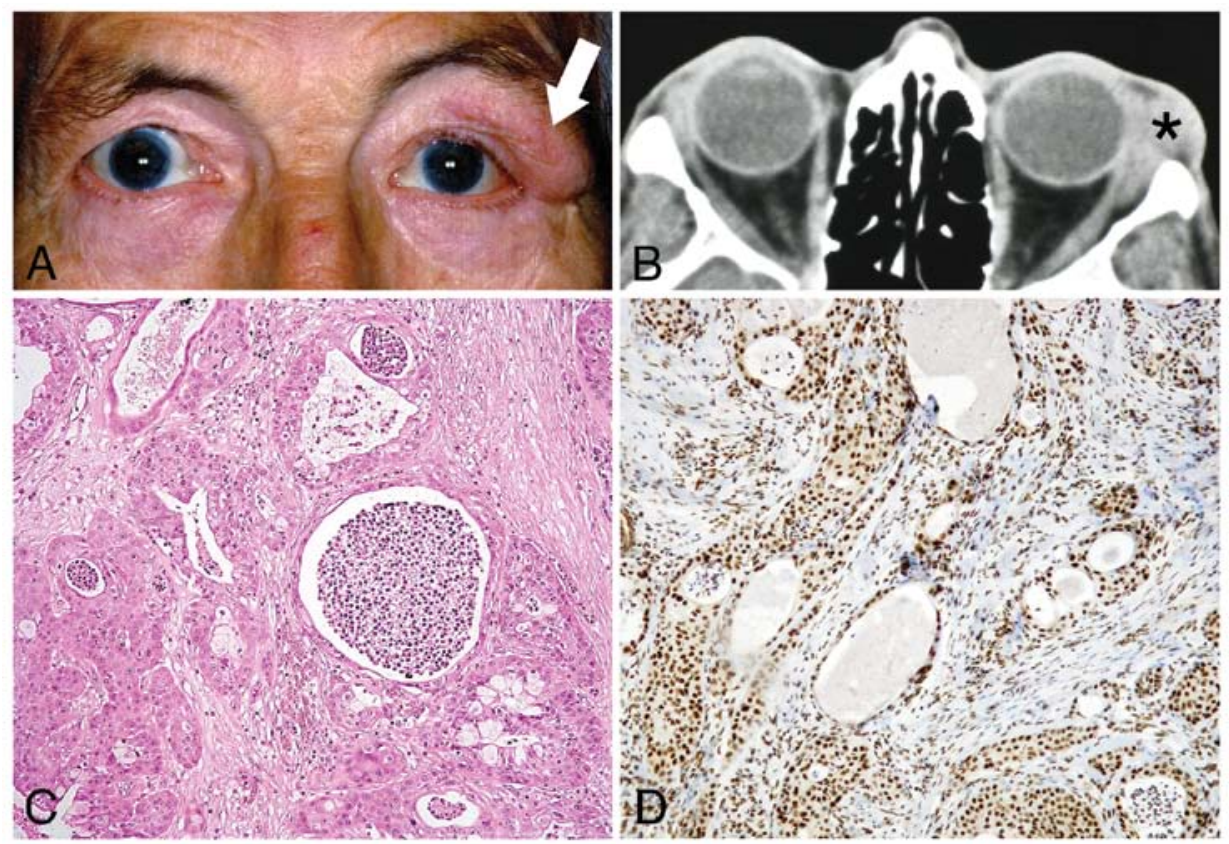

Figure 1. (A) Tumor of the left lacrimal gland indicated by an arrow. (B) Computed tomography scan showing the tumor at the site of the left lacrimal gland (asterisk). (C) Microphotograph of the tumor demonstrating cystic spaces lined by epidermoid, intermediate and mucus-producing cells (hematoxylin and eosin). (D) Immunostaining of the CRTC1-MAML2 fusion protein. Note the predominant nuclear staining of the tumor cells.

recurrence and the patient died of non-tumor related causes five years after surgery.

Tumor material. In addition to the lacrimal MEC, we had also access to formalin-fixed paraffin-embedded (FFPE) tumor material from 15 non-MEC lacrimal neoplasms, including 5 pleomorphic adenomas, 5 carcinoma ex pleomorphic adenomas and 5 adenoid cystic carcinomas. As a positive control we used cDNA from a previously described $t(11 ; 19)$ positive salivary MEC (11).

Histopathology and immunohistochemistry. The FFPE tumor tissue was sectioned and stained with haematoxylin and eosin, periodic acid-Schiff (PAS) and alcian blue according to standard protocols. Immunohistochemistry was performed with the Dako EnVision ${ }^{\mathrm{TM}}+$ System (Dako A/S, Glostrup, Denmark) using antibodies to the following antigens: cytokeratin (clone MN116, code no. M0821, Dako), vimentin (clone Vim 3B4, code no. M7020, Dako), epithelial membrane antigen (clone E29, code no. M613, Dako), glial fibrillary acidic protein (code no.Z0334, Dako), S-100 (code no.Z0311, Dako), smooth muscle actin (clone 1A4, code no. M0851, Dako), carcinoembryonic antigen (clone II-7, code no. M7072, Dako) and $\alpha$-fetoprotein (code no. A0008, Dako). The CRTC1-MAML2 fusion protein was detected using a polyclonal, fusion-specific antibody as previously described (11). Negative control sections were incubated identically except for the primary antibody, which was replaced by normal rabbit serum/mouse IgG.

RT-PCR and FISH analyses for detection of the CRTC1$M A M L 2$ gene fusion. Total RNA was extracted from three 20- $\mu \mathrm{m}$ FFPE tissue sections using the RNeasy FFPE Kit (Qiagen, Hilden, Germany). The RNA was subsequently converted to cDNA using the SuperScript ${ }^{\mathrm{TM}}$ First-Strand Synthesis System
(Invitrogen, Karlsruhe, Germany) with random hexamer primers as recommended by the manufacturer. As a control for intact RNA and cDNA, an RT-PCR reaction for expression of $A C T B$ was performed using the following primers: forward 5'-ATCA CCATTGGCAATGAGCG-3' and reverse 5'-TTGAAGGTAGT TTCGTGGAT-3' (amplification of a 98-bp product). The CRTC1-MAML2 fusion transcript was amplified by nested PCR. The first round PCR was carried out using the primers CRTC1-54F 5'-GAGAAGATCGCGCTGCAC-3' and MAML21855R 5'-CTTGCTGTTGGCAGGAGA-3' (amplification of a 150 -bp product) and the second round PCR was performed using the primers CRTC1-99F 5'-GCCTTCGAGGAGGTCA TGA-3' and MAML2-1834R 5'-GGTTAACTACCTGTTTTCT TTTCAAGG-3' (amplification of a 85-bp product). The PCR product was subsequently gel-purified and sequenced using an ABI PRISM 310 Genetic Analyzer (Applied Biosystems, Foster City, CA).

Fluorescence in situ hybridization (FISH) analysis of FFPE tissue sections $(3 \mu \mathrm{m})$ was performed using the ZytoLight ${ }^{\circledR}$ SPEC MAML2 Dual Color Break Apart Probe (ZytoVision, Bremerhaven, Germany). The protocols for pretreatment and hybridization were essentially as recommended by the manufacturer. After being washed, the sections were counterstained with 40,60-diamidino-20-phenylindole dihydrochloride (DAPI). Slides were examined in a Zeiss Axioplan 2 fluorescence microscope (Carl Zeiss, Oberkochen, Germany) equipped with appropriate filter sets.

\section{Results}

Histopathological features. Macroscopic examination of the surgical specimen revealed a circumscribed tumor with grayish-white lobulated cut surfaces. Microscopically, the tumor was surrounded by a pseudocapsule and composed 


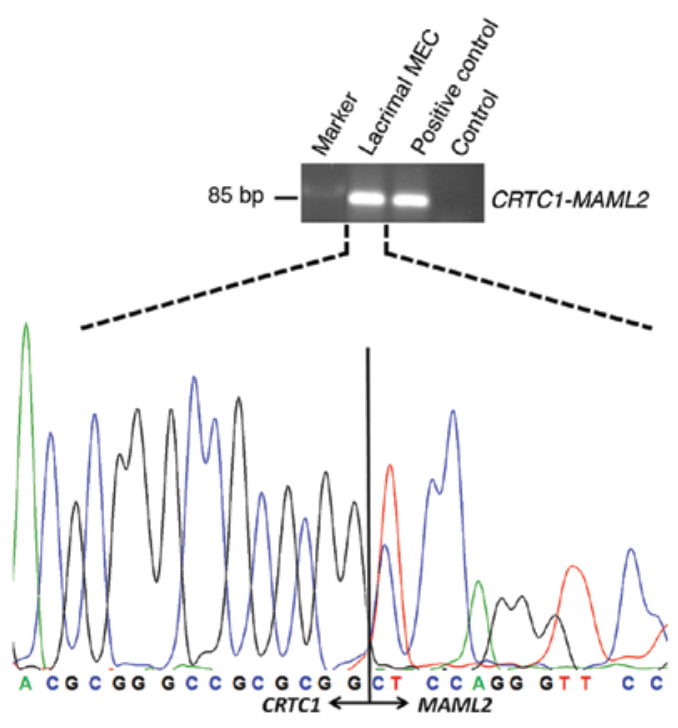

Figure 2. Detection of the CRTC1-MAML2 fusion transcript in lacrimal MEC. RT-PCR analysis revealed an 85-bp fragment in the lacrimal MEC as well as in a t(11;19)-positive salivary MEC (positive control). Nucleotide sequence analysis of the PCR-product generated from the lacrimal MEC confirmed that it indeed corresponded to a chimeric transcript in which exon 1 of $C R T C 1$ is linked to exon 2 of MAML2. The control lane represents PCR-reactions with gene-specific primers but without cDNA template. The size of the amplified fragment is indicated.

of cystic structures lined by epidermoid, intermediate and mucous-producing cells (Fig. 1C). The epidermoid cells had large nuclei and a strongly eosinophilic cytoplasm without signs of keratinisation. The mucous-producing cells had prominent nucleoli and light eosinophilic cytoplasms. Occasional clear cells were observed. The mucous stained positive with PAS and alcian blue. The degree of anaplasia was low and only a few mitoses were observed. There were no signs of neural invasion or necrosis. The epidermoid tumor cells stained positive for cytokeratin and epithelial membrane antigen whereas the stromal cells were positive for vimentin and smooth muscle actin. Neither the tumor cells nor the stromal cells expressed S-100, $\alpha$-fetoprotein, carcinoembryonic antigen or glial fibrillary acidic protein. Taken together, these findings are consistent with the diagnosis of a low-grade MEC of the lacrimal gland.

Expression of CRTC1-MAML2 fusion transcript and protein. RT-PCR analysis of cDNA prepared from the lacrimal MEC revealed expression of an 85-bp CRTC1-MAML2 fusion transcript (Fig. 2). This transcript was also detected in a $t(11 ; 19)$ translocation-positive salivary MEC but not in 15 non-MEC lacrimal gland neoplasms (data not shown). The identity of the PCR-product was confirmed by nucleotide sequence analysis, which demonstrated a fusion between exon 1 of $C R T C l$ and exon 2 of MAML2 (Fig. 2). FISH analysis of FFPE sections of the tumor using a $M A M L 2$-specific dual color break apart probe, revealed rearrangements of $M A M L 2$, consistent with a CRTC1-MAML2 gene fusion in the majority of tumor cells analyzed (data not shown). In contrast, rearrangements of $M A M L 2$ were not observed in any of the intermingled and surrounding stromal cells.
The expression of the CRTC1-MAML2 fusion protein was also studied by immunohistochemistry using a custommade polyclonal CRTC1-MAML2 antibody. Consistent with the FISH-results, we observed distinct nuclear staining in the majority of tumor cells, including epidermoid, intermediate, and mucous producing cells (Fig. 1D).

\section{Discussion}

Epithelial tumors of the lacrimal gland are rare and MEC only constitutes a few percent of these; the most common lacrimal gland neoplasms being pleomorphic adenomas and adenoid cystic carcinomas. Here we report on a rare case of lacrimal MEC in a 73-year-old man. The tumor had a microscopic appearance consistent with a low-grade MEC. There were no signs of recurrence or metastases during a 5-year follow-up, after which the patient died of non-tumor related causes.

Since we and others previously have shown that MECs originating from the major and minor salivary glands, lung, thyroid, and cervix are characterized by a recurrent $C R T C 1$ MAML2 gene fusion $(6,7,11,14,15)$, we decided to investigate whether this fusion also occurs in MEC of the lacrimal gland. Using RT-PCR and FISH we demonstrated that the present case of lacrimal MEC indeed was fusion-positive. By immunohistochemistry we also showed that the majority of tumor cells, including epidermoid, intermediate and mucous producing cells, expressed the CRTC1-MAML2 fusion protein. In contrast, 15 non-MEC lacrimal tumors were fusionnegative, indicating that CRTC1-MAML2 is a MEC-specific fusion also in the lacrimal gland. These findings, which are in agreement with our previous observations in salivary MEC (11), further strengthens the notion that the CRTC1-MAML2 fusion is an early genetic event in the pathogenesis of MEC. Previous studies have also shown that sustained expression of the fusion is necessary for growth of MEC tumor cells (16). Taken together, these observations indicate that the CRTC1$M A M L 2$ fusion is an oncogenic driver mutation in MEC and as such the fusion may also be a highly relevant therapeutic target. An identical CRTC1-MAML2 gene fusion has also been identified in metaplastic variants of Warthins tumor and in clear cell hidradenomas of the skin $(7,14,17)$, thus broadening the spectrum of neoplasms associated with this gene fusion.

The present case shows that lacrimal MEC is not only clinically and morphologically but also genetically identical to MECs originating from other exocrine glands. The frequency of the CRTC1-MAML2 fusion in lacrimal MEC remains unknown. However, previous studies have shown that up to approximately $80 \%$ of salivary MECs are fusion-positive and that the fusion preferentially occurs in low-grade tumors with an excellent prognosis (11-14). This is in agreement with the present case which was a low-grade, fusion-positive MEC with favorable prognosis. Based on these findings we suggest that the CRTC1-MAML2 fusion will be a useful diagnostic and prognostic biomarker also for lacrimal MEC.

The results of the present study are in line with the recently suggested concept that MEC may be divided into several clinically, morphologically and genetically different subgroups, that is i) fusion-positive low- and intermediate-grade tumors with mainly favorable outcome, ii) fusion-positive high-grade tumors with unfavorable prognosis, and iii) fusion-negative 
tumors that may be more appropriately categorized as another tumor type, such as for example adenosquamous carcinoma $(11,13)$. Further studies of MEC and MEC-like tumors of the lacrimal gland are necessary to find out how they fit into this scheme.

\section{Acknowledgements}

The study was supported by The Danish Eye Research Foundation, the Danish Eye Health Society, The Danish Cancer Society, Synoptik-Fonden, Købmand Kristjan Kjær and wife Magrethe Kjær's Foundation, Kleinsmed Svend Helge Arvid Schrøder and wife Ketty Lydia Larsen Schrøder's Foundation, DMSc Alfred Helsted and wife DMSc Eli Møller's Foundation, Engineer August Fredrik Wedell Erichsen's Foundation, the Swedish Cancer Society and BioCARE - a National Strategic Research Program at University of Gothenburg.

\section{References}

1. Eviatar JA and Hornblass A: Mucoepidermoid carcinoma of the lacrimal gland: 25 cases and a review and update of the literature. Ophthal Plast Reconstr Surg 9: 170-181, 1993.

2. Sofinski SJ, Brown BZ, Rao N and Wan WL: Mucoepidermoid carcinoma of the lacrimal gland. Case report and review of the literature. Ophthal Plast Reconstr Surg 2: 147-151, 1986.

3. Font RL, Croxatto JO and Rao NA: Tumors of the lacrimal gland. In: Tumors of the Eye and Ocular Adnexa. 1st edition. Silverberg SG (ed). American Registry of Pathology/Armed Forces Institute of Pathology, Washington, pp223-246, 2006.

4. Goode RK and El Naggar AK: Mucoepidermoid carcinoma. In: World Health Organization Classification of Tumors. Pathology and Genetics Head and Neck Tumours. 1st edition. Barnes L, Eveson J, Reichart P and Sidransky D (eds). IARC Press, Lyon, pp219-220, 2005.

5. Nordkvist A, Gustafsson H, Juberg-Ode M and Stenman G Recurrent rearrangements of 11q14-22 in mucoepidermoid carcinoma. Cancer Genet Cytogenet 74: 77-83, 1994.

6. Tonon G, Modi S, Wu L, Kubo A, Coxon AB, Komiya T, O'Neil K, Stover K, El-Naggar A, Griffin JD, Kirsch IR and Kaye FJ: $\mathrm{t}(11 ; 19)(\mathrm{q} 21 ; \mathrm{p} 13)$ translocation in mucoepidermoid carcinoma creates a novel fusion product that disrupts a Notch signaling pathway. Nat Genet 33: 208-213, 2003.
7. Enlund F, Behboudi A, Andren Y, Öberg C, Lendahl U, Mark J and Stenman G: Altered Notch signaling resulting from expression of a WAMTP1-MAML2 gene fusion in mucoepidermoid carcinomas and benign Warthin's tumors. Exp Cell Res 292: 21-28, 2004.

8. Stenman G: Fusion oncogenes and tumor type specificity insights from salivary gland tumors. Semin Cancer Biol 15: 224-235, 2005

9. Coxon A, Rozenblum E, Park YS, Joshi N, Tsurutani J, Dennis PA, Kirsch IR and Kaye FJ: Mect1-Maml2 fusion oncogene linked to the aberrant activation of cyclic AMP/CREB regulated genes. Cancer Res 65: 7137-7144, 2005.

10. Wu L, Liu J, Gao P, Nakamura M, Cao Y, Shen H and Griffin JD: Transforming activity of MECT1-MAML2 fusion oncoprotein is mediated by constitutive CREB activation. EMBO J 24: 2391-2402, 2005

11. Behboudi A, Enlund F, Winnes M, Andren Y, Nordkvist A, Leivo I, Flaberg E, Szekely L, Makitie A, Grenman R, Mark J and Stenman G: Molecular classification of mucoepidermoid carcinomas-prognostic significance of the MECT1-MAML2 fusion oncogene. Genes Chromosomes Cancer 45: 470-481, 2006.

12. Okabe M, Miyabe S, Nagatsuka H, Terada A, Hanai N, Yokoi M, Shimozato K, Eimoto T, Nakamura S, Nagai N, Hasegawa Y and Inagaki H: MECT1-MAML2 fusion transcript defines a favorable subset of mucoepidermoid carcinoma. Clin Cancer Res 12: 3902-3907, 2006.

13. Seethala RR, Dacic S, Cieply K, Kelly LM and Nikiforova MN: A reappraisal of the MECT1/MAML2 translocation in salivary mucoepidermoid carcinomas. Am J Surg Pathol 34: 1106-1121, 2010.

14. Tirado Y, Williams MD, Hanna EY, Kaye FJ, Batsakis JG and El-Naggar AK: CRTC1/MAML2 fusion transcript in high grade mucoepidermoid carcinomas of salivary and thyroid glands and Warthin's tumors: implications for histogenesis and biologic behavior. Genes Chromosomes Cancer 46: 708-715, 2007.

15. Lennerz JK, Perry A, Mills JC, Huettner PC and Pfeifer JD: Mucoepidermoid carcinoma of the cervix: another tumor with the $\mathrm{t}(11 ; 19)$-associated CRTC1-MAML2 gene fusion. Am J Surg Pathol 33: 835-843, 2009.

16. Komiya T, Park Y, Modi S, Coxon AB, Oh H and Kaye FJ: Sustained expression of Mect1-Maml2 is essential for tumor cell growth in salivary gland cancers carrying the $t(11 ; 19)$ translocation. Oncogene 25: 6128-6132, 2006.

17. Winnes M, Molne L, Suurkula M, Andren Y, Persson F, Enlund F and Stenman G: Frequent fusion of the CRTC1 and MAML2 genes in clear cell variants of cutaneous hidradenomas. Genes Chromosomes Cancer 46: 559-563, 2007. 